\title{
Slow breathing influences cardiac autonomic responses to postural maneuver Slow breathing and HRV
}

\author{
Giovanna Ana de Paula Vidigal a , Bruna S. Tavares a , David M. Garner ${ }^{\mathrm{b}}$, Andrey A. Porto ${ }^{\mathrm{c}}$, \\ Luiz Carlos de Abreu ${ }^{\mathrm{d}}$, Celso Ferreira ${ }^{\mathrm{c}}$, Vitor E. Valenti ${ }^{\mathrm{e}}$ * \\ a Centro de Estudos do Sistema Nervoso Autônomo (CESNA), Departamento de Fisioterapia e Terapia Ocupacional, Faculdade de Filosofia e Ciências, UNESP, \\ Marília, SP, Brazil \\ ${ }^{\mathrm{b}}$ Cardiorespiratory Research Group, Department of Biological and Medical Sciences, Faculty of Health and Life Sciences, Oxford Brookes University, Gipsy \\ Lane, Oxford OX3 OBP, United Kingdom \\ c Disciplina de Cardiologia, Departamento de Medicina, UNIFESP, São Paulo, SP, Brazil \\ ${ }^{d}$ Department of Environmental Health, Harvard Medical School of Public Health, Boston, MA, United States \\ e Centro de Estudos do Sistema Nervoso Autônomo (CESNA), Programa de Pós-Graduação em Fisioterapia, Faculdade de Ciências e Tecnologia, UNESP, \\ Presidente Prudente, SP, Brazil
}

\section{A R T I C L E I N F O}

\section{Article history:}

Received 13 November 2015

Accepted 26 November 2015

\section{Keywords:}

Cardiovascular system

Autonomic nervous system

Respiration

Complementary therapy

\begin{abstract}
A B S T R A C T
Chronic slow breathing has been reported to improve Heart Rate Variability (HRV) in patients with cardiovascular disorders. However, it is not clear regarding its acute effects on HRV responses on autonomic analysis. We evaluated the acute effects of slow breathing on cardiac autonomic responses to postural change manoeuvre (PCM). The study was conducted on 21 healthy male students aged between 18 and 35 years old. In the control protocol, the volunteer remained at rest seated for 15 min under spontaneous breathing and quickly stood up within $3 \mathrm{~s}$ and remained standing for $15 \mathrm{~min}$. In the slow breathing protocol, the volunteer remained at rest seated for 10 min under spontaneous breath, then performed slow breathing for 5 min and rapidly stood up within $3 \mathrm{~s}$ and remained standing for 15 min. Slow breathing intensified cardiac autonomic responses to postural maneuver.
\end{abstract}

(c) 2016 Elsevier Ltd. All rights reserved.

\section{Introduction}

Slow breathing has gained consideration because the literature reported positive physiological effects, including blood pressure reduction and improvement in chronic heart failure [1-3]. Reduction in the respiratory rate also acutely reduced anxiety and, it was considered sufficient to control physiological arousal during stressful conditions in musicians [4].

The effects of slow breathing include beneficial changes in the Autonomic Nervous System (ANS) [5]. It was demonstrated that slow breathing increases parasympathetic cardiac modulation and reduces sympathetic cardiac control [6]. In this manner, Heart Rate Variability (HRV) $[7,8]$ is a simple and non-invasive method that analyzes cardiac autonomic regulation. It measures the fluctuation

* Corresponding author. Departamento de Fonoaudiologia, Av. Hygino Muzzi Filho, 737, 17.525-000, Marília, SP, Brazil.

E-mail address: vitor.valenti@marilia.unesp.br (V.E. Valenti). of the intervals between consecutive heart beats (RR intervals) [9].

Previous studies have illustrated increases in HRV caused by chronic guided breathing sessions in hypertensive subjects. These studies support slow breathing as a complementary and alternative intervention for cardiovascular disorders $[10,11]$.

Autonomic tests are applied to evaluate the adequate function of the ANS. A standard test applied in the clinical daily routine is the postural change maneuver (PCM). This test is based on the measurement of heart rate reflex changes in response to postural change stimulation [12].

A further study demonstrated that slow breathing training decreased baseline blood pressure and declined the pressor response to handgrip exercise [13]. Nonetheless, the acute effects of slow breathing on HRV responses to different autonomic tests are unclear. Additionally, non-pharmacological intervention is of assistance to add new elements in alternative and complementary therapies. In this situation, we investigated the acute effects of slow breathing on cardiac autonomic responses to PCM. 


\section{Methods}

\subsection{Study population}

The subjects participating in the study were 21 healthy male students - all non-smokers, aged between 18 and 35 years old. All volunteers were informed about the procedures and the objectives of the study and gave written informed consent. All study procedures were approved by the Ethics Committee in Research of our Institution (No. 2014-953), and were in accordance with Resolution 196/96 National Health 10/10/1996.

\subsection{Non-inclusion criteria}

We excluded subjects under the following conditions: Body Mass Index (BMI) > $35 \mathrm{~kg} / \mathrm{m}^{2}$; systolic blood pressure $(\mathrm{SBP})>140 \mathrm{mmHg}$ or diastolic blood pressure (DBP) $>90 \mathrm{mmHg}$ (at rest); cardiovascular, respiratory, endocrine and reported neurological disorders that did not allow the volunteers to perform the procedures. Subjects under medication that influence the ANS were excluded.

\subsection{Initial evaluation}

Baseline information collected included age, gender, mass, height and Body Mass Index (BMI). Mass was determined using a digital scale (W 200/5, Welmy, Sao Paulo, Brazil) with a precision of $0.1 \mathrm{~kg}$. Height was determined using a stadiometer (ES 2020, Sanny, Sao Paulo, Brazil) with a precision of $0.1 \mathrm{~cm}$ and $220 \mathrm{~cm}$ of extension. BMI was calculated as weight/height [2], with weight in kilograms and height in meters.

\subsection{HRV analysis}

HRV was analyzed according to instructions from the Task Force guidelines [7]. Instantaneous RR intervals ( $\mathrm{RRi}$ ) were recorded with a digital telemetry system, consisting of a transmitter placed on the patient's chest and a heart rate monitor (Polar ${ }^{\circledR}$ RS800CX; Polar Electro Oy, Kempele, Finland). This system detects ventricular depolarization, corresponding to the $\mathrm{R}$ wave on the electrocardiogram. This was achieved at a sampling rate of $500 \mathrm{~Hz}$. It had been previously validated [14] and further downloaded to the Polar Precision Performance program (v.3.0, Polar Electro, Finland). The software enabled the visualization of heart rate and the extraction of a cardiac period (RR interval) file in "txt" format. Subsequent digital filtering complemented with manual filtering for the elimination of premature ectopic beats and artefacts, 256 RR intervals were applied for the data analysis. Only series with sinus rhythm greater than $95 \%$ were included in the study.

\subsection{Time and frequency domain indices of $H R V$}

For HRV analysis in the frequency domain we applied the spectral components of low frequency (LF: $0.04-0.15 \mathrm{~Hz}$ ) and high frequency (HF: $0.15-0.40 \mathrm{~Hz}$ ) in absolute (ms [2]) and in normalized units. The spectral analysis was calculated with the Fast Fourier Transform (FFT) algorithm [15].

Time domain analysis was accomplished through the SDNN (average standard deviation of normal RR intervals), pNN50 (percentage of adjacent $R R$ intervals lasting more difference than $50 \mathrm{~ms}$ ), RMSSD (square root of the average square differences between normal adjacent RR intervals) and SDNN/RMSSD ratio [16]. For analysis of linear indices in the frequency and time domain we applied the Kubios HRV ${ }^{\mathbb{R}}$ analysis software [17].

\subsection{Slow breathing protocol}

The slow breathing protocol was based on the literature which emphasized cycles with 10-12 s duration, corresponding to a breathing rate of 5-6 cycles per minute [18]. In this modus operandi the volunteers performed about 6 cycles per minute each with $10 \mathrm{~s}$ duration $(0.1 \mathrm{~Hz})$ for $5 \mathrm{~min}$. The researcher guided the volunteer's breathing with a metronome. The volunteers were instructed to perform deep, but slow inspirations, and similar expirations with lung volumes ranging from the total lung volume to residual volume.

\subsection{Experimental protocols}

Data collection was undertaken in the same sound-proofed laboratory for all volunteers with the temperature between $20{ }^{\circ} \mathrm{C}$ and $26^{\circ} \mathrm{C}$; and relative humidity between $40 \%$ and $60 \%$. Volunteers were instructed not to drink alcohol and/or caffeine for $24 \mathrm{~h}$ before evaluation and $24 \mathrm{~h}$ with no extenuous exercise, with a light meal at least $2 \mathrm{~h}$ before conducting tests. Datasets were collected on an individual basis, between 18:00 $\mathrm{h}$ and 21:00 $\mathrm{h}$ to standardize the circadian cycle. All procedures necessary for the data collection were explained on an individual basis and the subjects were instructed to remain at rest and avoid conversation during the data collection.

In the slow breathing protocol, the subject remained at rest seated for $10 \mathrm{~min}$ under spontaneous breathing. After $10 \mathrm{~min}$, the volunteers performed slow breathing for $5 \mathrm{~min}$ and promptly stood up from a seated position within $3 \mathrm{~s}$ according to verbal command and remained standing for $15 \mathrm{~min}$. In the control protocol, the subject remained at rest seated for 15 min under spontaneous breathing (Table 1). The sequence of the protocols was randomized.

\subsection{Statistical analysis}

Standard statistical methods were applied for the calculation of means and standard deviations. Normal Gaussian distribution of

Table 1

Experimental protocols.

\begin{tabular}{|c|c|c|c|c|c|c|}
\hline Control & $10 \mathrm{~min}$ & $10-15 \mathrm{~min}$ & $15 \mathrm{~min}$ & $15-20$ & $20-25$ & $25-30$ \\
\hline & $\begin{array}{l}\text { HRV recording under } \\
\text { spontaneous breathing at } \\
\text { seated rest. }\end{array}$ & $\begin{array}{l}\text { HRV recording under } \\
\text { spontaneous breathing at } \\
\text { seated rest. }\end{array}$ & $\begin{array}{l}\text { Change from } \\
\text { seated to } \\
\text { standing. }\end{array}$ & $\begin{array}{l}\text { HRV recording at } \\
\text { standing under } \\
\text { spontaneous breathing. }\end{array}$ & $\begin{array}{l}\text { HRV recording at } \\
\text { standing under } \\
\text { spontaneous breathing. }\end{array}$ & $\begin{array}{l}\text { HRV recording at } \\
\text { standing under } \\
\text { spontaneous breathing. }\end{array}$ \\
\hline \multirow{2}{*}{$\begin{array}{l}\text { Paced breathing } \\
\text { protocol }\end{array}$} & $10 \mathrm{~min}$ & $10-15 \mathrm{~min}$ & $15 \mathrm{~min}$ & $15-20$ & $20-25$ & $25-30$ \\
\hline & $\begin{array}{l}\text { HRV recording under } \\
\text { spontaneous breathing at } \\
\text { seated rest. }\end{array}$ & $\begin{array}{l}\text { Slow breathing at seated } \\
\text { rest. }\end{array}$ & $\begin{array}{l}\text { Change from } \\
\text { seated to } \\
\text { standing. }\end{array}$ & $\begin{array}{l}\text { HRV recording at } \\
\text { standing under } \\
\text { spontaneous breathing. }\end{array}$ & $\begin{array}{l}\text { HRV recording at } \\
\text { standing under } \\
\text { spontaneous breathing. }\end{array}$ & $\begin{array}{l}\text { HRV recording at } \\
\text { standing under } \\
\text { spontaneous breathing. }\end{array}$ \\
\hline
\end{tabular}


Table 2

Baseline diastolic (DAP) and systolic arterial pressure (SAP), heart rate, mean RR interval, weight, height and body mass index (BMI) of the volunteers. Mean \pm standard-deviation. m: meters; ms: millisecond; kg: kilograms; bpm: beats per minute; mmHg: millimeters of mercury.

\begin{tabular}{lc}
\hline Variable & Value \\
\hline Age $($ years $)$ & $21.5 \pm 2.5$ \\
Height $(\mathrm{m})$ & $1.78 \pm 0.1$ \\
Weight $(\mathrm{kg})$ & $78.3 \pm 17.9$ \\
BMI $\left(\mathrm{kg} / \mathrm{m}^{2}\right)$ & $24.5 \pm 4.9$ \\
Heart Rate $(\mathrm{bpm})$ & $77.8 \pm 10$ \\
Mean RR $(\mathrm{ms})$ & $788.108 \pm 61$ \\
SAP $(\mathrm{mmHg})$ & $118.3 \pm 10.9$ \\
DAP $(\mathrm{mmHg})$ & $72.9 \pm 11$ \\
\hline
\end{tabular}

the data was verified by the Shapiro-Wilk goodness-of-fit test ( $\mathrm{z}$ value $>1.0$ ). HRV was analyzed in the last 5 min of seated rest under spontaneous breathing, 0-5 $\mathrm{min}, 5-10 \mathrm{~min}$ and $10-15 \mathrm{~min}$ at standing under spontaneous breathing. For parametric distributions we applied paired one-way ANOVA followed by Bonferroni posttest. For non-parametric distributions we applied the Newman-Keuls posttest. Differences were considered significant when the probability of a Type I error was less than $5 \%(p<0.05)$. We used the Biostat ${ }^{\circledR}$ Software 2009 Professional v5.8.4 (Analysis Soft, Walnut, California, USA).
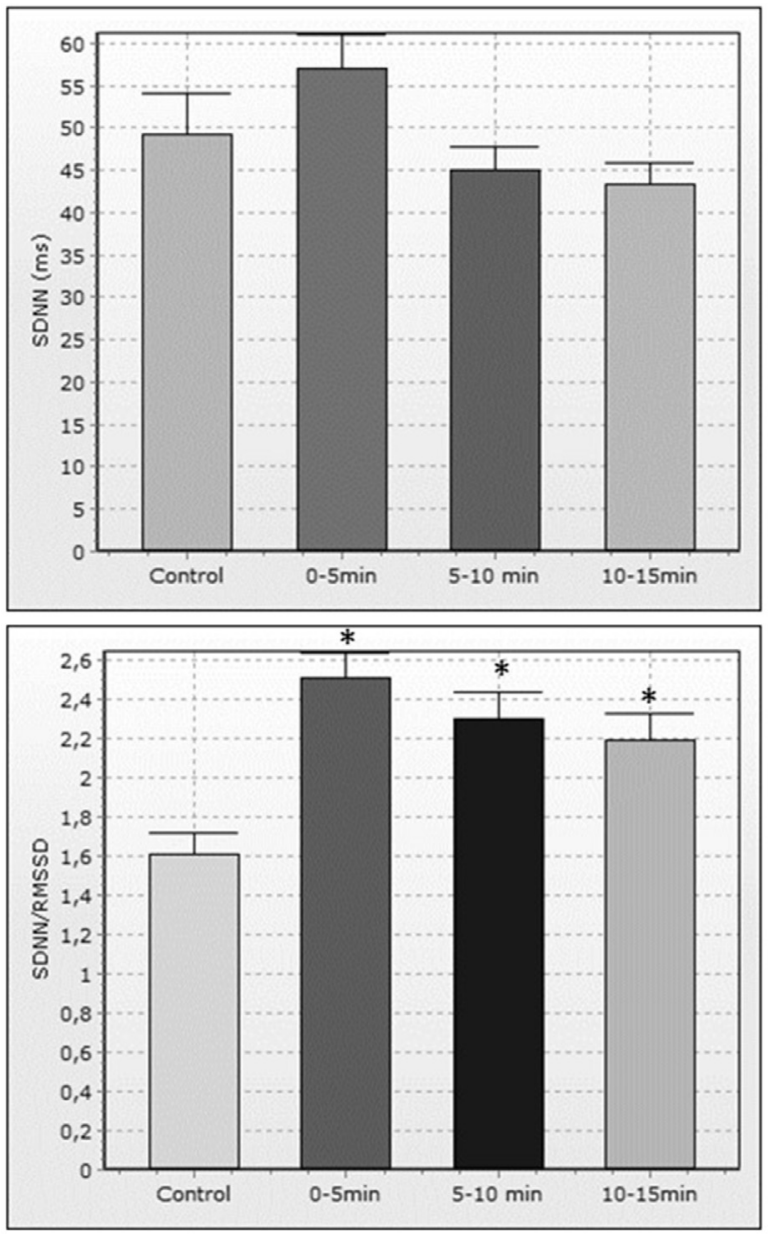

\section{Results}

We observe data in Table 2 regarding baseline systolic arterial pressure (SAP) and diastolic arterial pressure (DAP), heart rate and mean RR interval, age, height, body mass and Body Mass Index (BMI).

Fig. 1 illustrates the time domain indices of HRV for the control protocol (at seated control) and during the 15 min after the volunteer stood up. We observed that the RMSSD and pNN50 reduced 10-15 min after the subjects stood up compared to control (seated vs. 10-15 $\mathrm{min}$ ). The SDNN/RMSSD ratio was increased during the $15 \mathrm{~min}$ after the subjects stood up compared to control (seated vs. 0-15 $\mathrm{min}$ ).

Regarding spectral analysis of HRV in the control protocol, the LF (nu) increased and HF (nu) declined 0-5 min and 10-15 min after the subjects stood up compared to control (Fig. 2).

Regarding the slow breathing protocol, the SDNN index increased 0-5 min after the subjects stood up compared to control. The RMSSD and pNN50 lowered 5-10 min and 10-15 min after the subjects stood up compared to control, whereas the SDNN/RMSSD index improved 0-15 min after the subjects stood up compared to control (Fig. 3).

Concerning the frequency domain analysis in the slow breathing protocol, the $\mathrm{LF} / \mathrm{HF}$ ratio increased $0-5 \mathrm{~min}$ after the subjects stood up compared to control, the LF (nu) also increased 0-15 min after
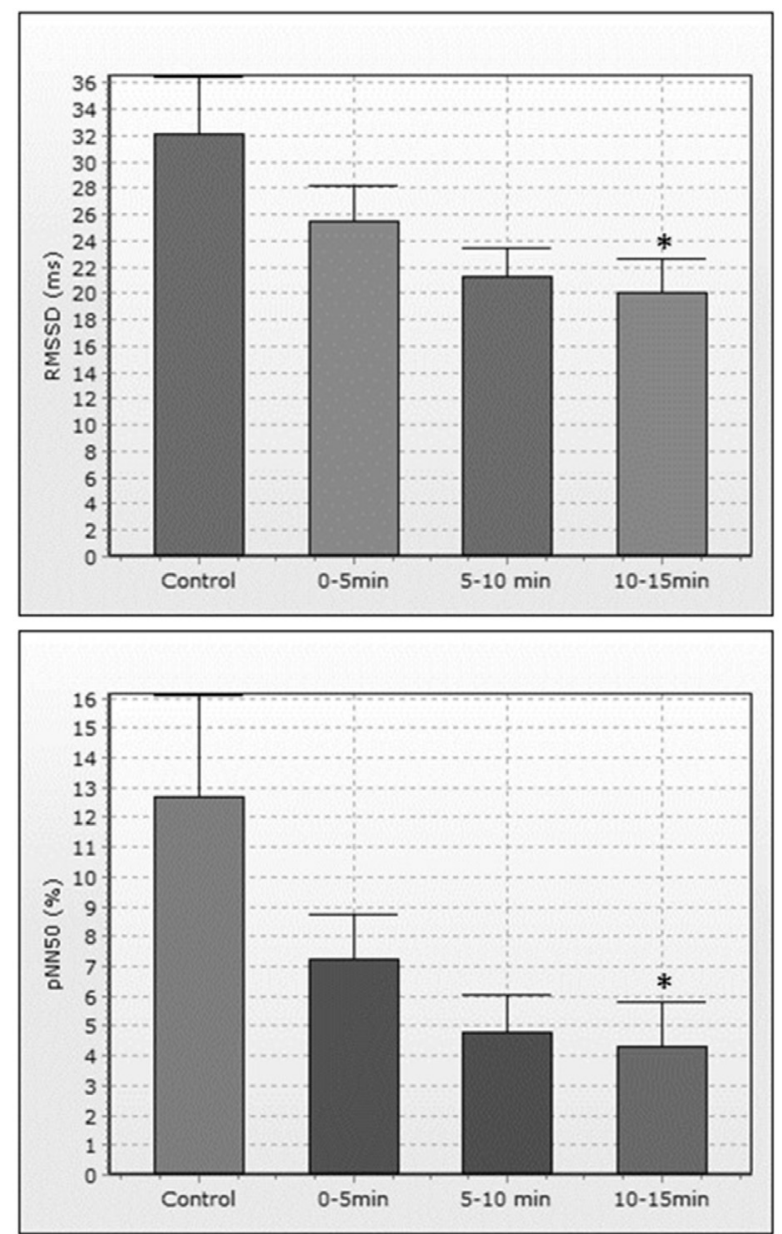

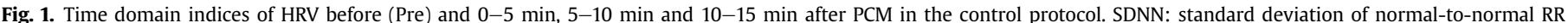

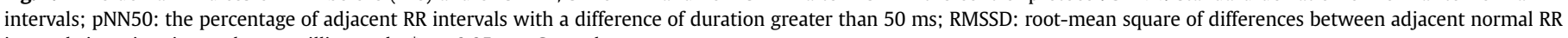
intervals in a time interval; ms: milliseconds. ${ }^{*} \mathrm{p}<0.05$ : vs. Control. 

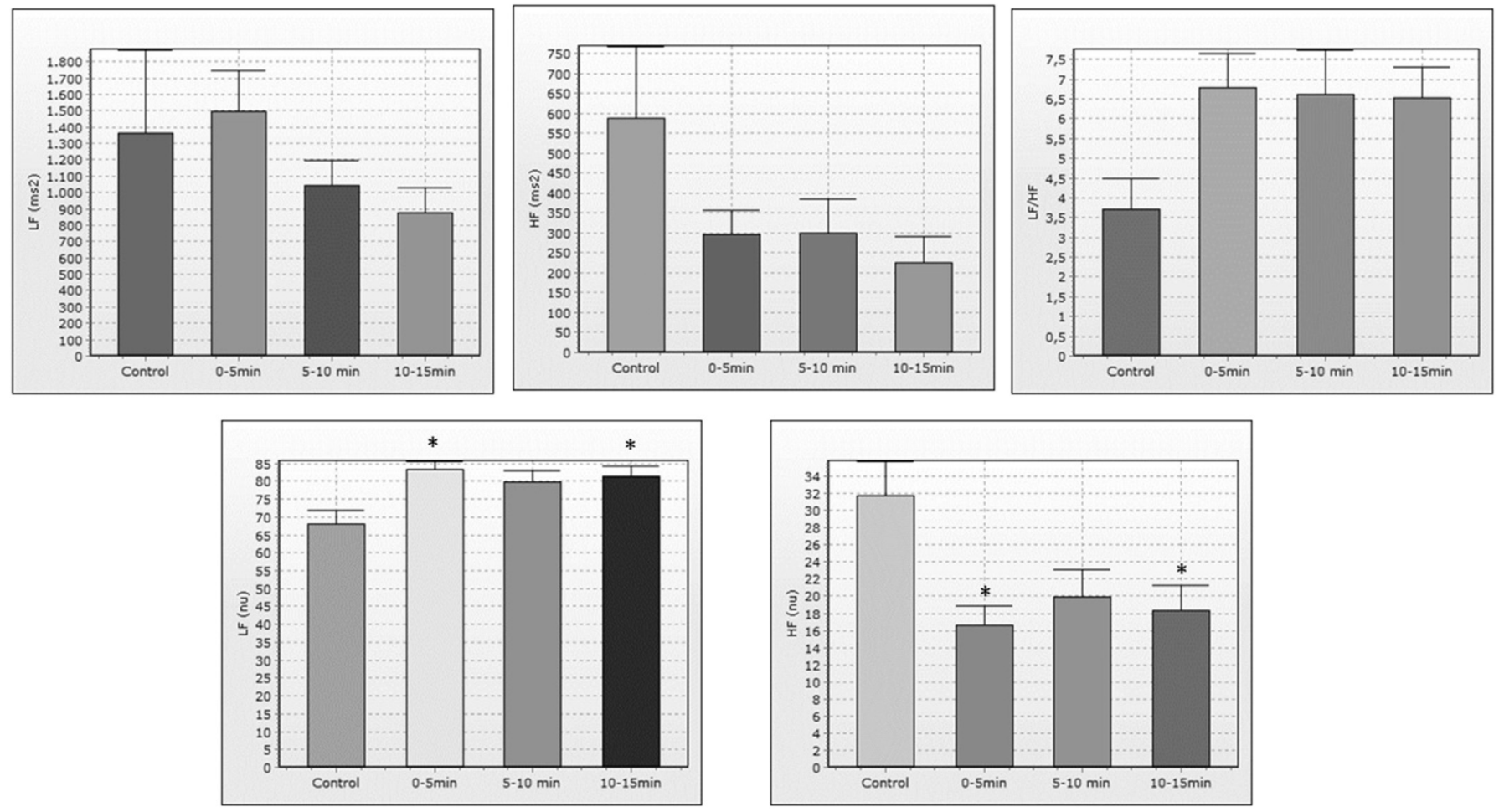

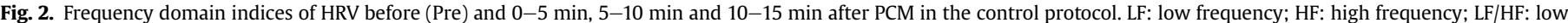
frequency/high frequency ratio; ms: milliseconds. ${ }^{*} \mathrm{p}<0.05$ : vs. Control.
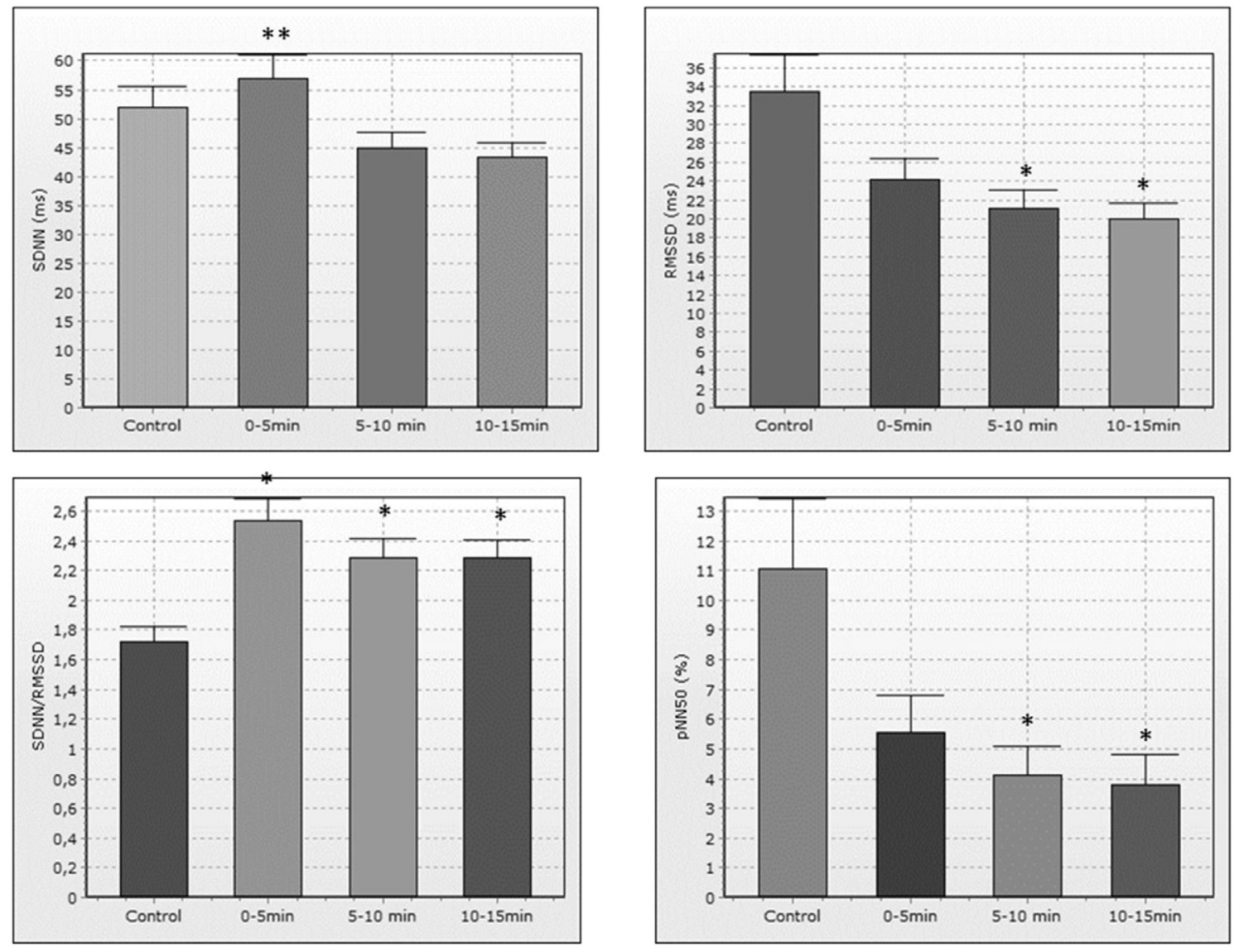

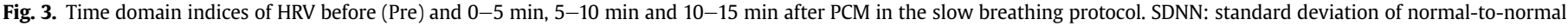

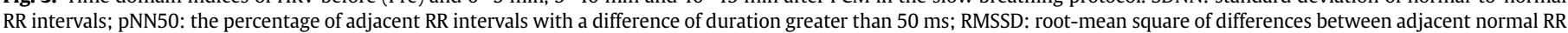
intervals in a time interval; ms: milliseconds. ${ }^{*} \mathrm{p}<0.05$ : vs. Control; ${ }^{* *} \mathrm{p}<0.05$ : vs. $10-15$ min. 

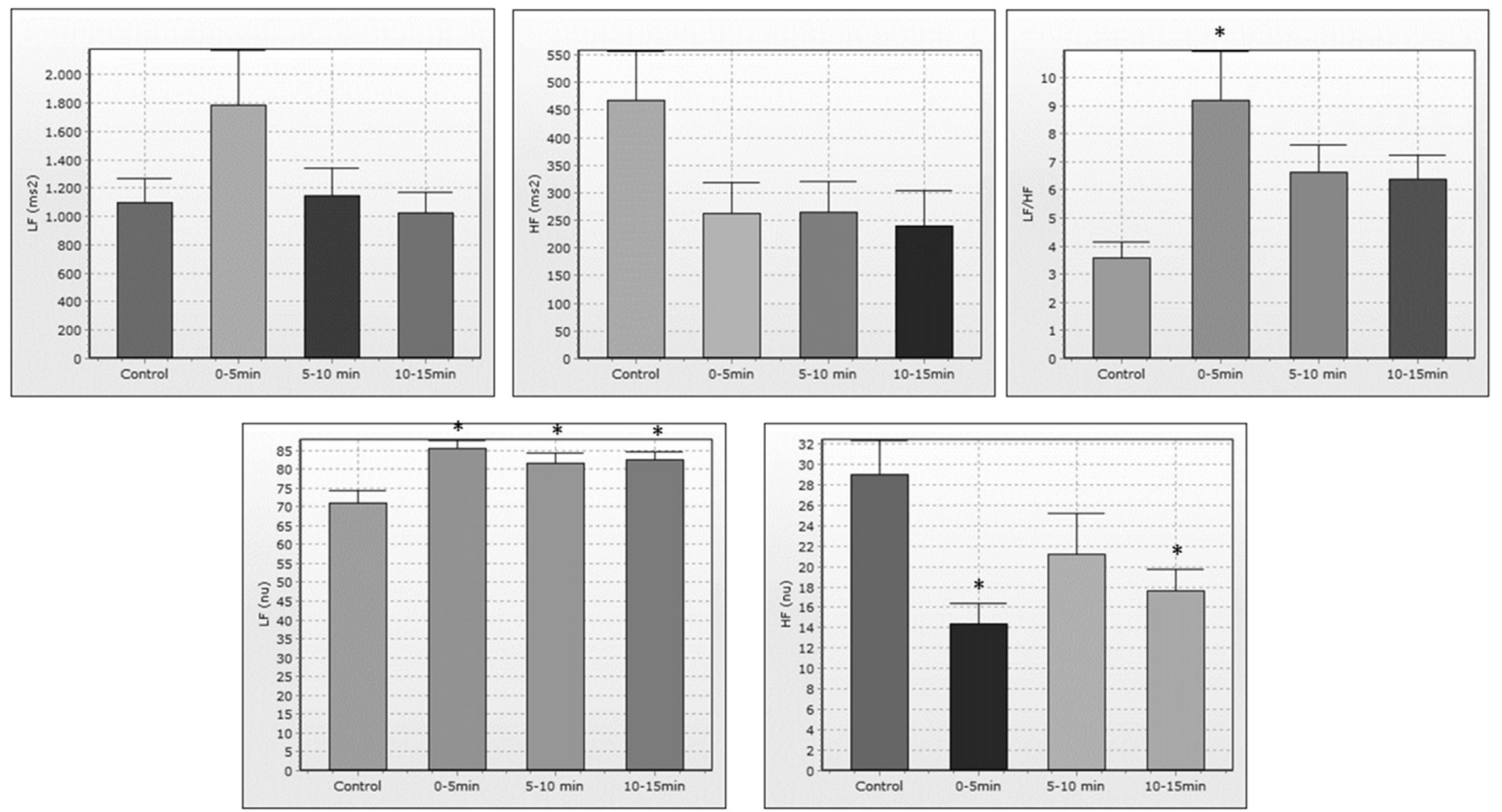

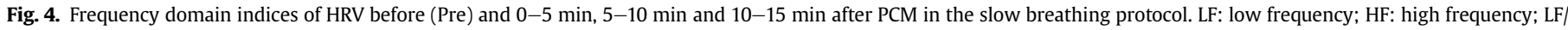
HF: low frequency/high frequency ratio; ms: milliseconds. ${ }^{*} \mathrm{p}<0.05$ : vs. Control.

the subjects stood up compared to control and the HF (nu) decreased $0-5$ and $10-15$ min after the subjects stood up compared to control (Fig. 4).

\section{Discussion}

At this point we investigated the acute effects of slow breathing on cardiac autonomic responses elicited by varying from seated to orthostatis. Standard cardiac autonomic mechanisms involved in the PCM were evaluated in the control protocol that included spontaneous breathing instead of slow breathing. We noted more intense responses of time and frequency domain indices of HRV in the slow breathing protocol, indicating that it intensified cardiac autonomic reactivity.

Here the slow breathing protocol was based on 6 cycles per minute. Breathing patterns of approximately 6 cycles per minute coincides with spontaneous Mayer wave frequency, increasing cardiac oscillation by synchronizing sympathetic and vagal systems [19].

There is a synchrony between breathing and cardiac cycle, this phenomenon is called respiratory sinus arrhythmia [20]. Acute changes in breath causes alterations in heart rate and blood pressure. During inspiration heart rate increases due to the vagal withdrawal and during expiration the parasympathetic cardiac regulation rises over the sinus node and the heart rate decreases [21]. The slow breathing influences the cardiac regulation because it is associated with increased baroreflex sensitivity, HRV and cardiac input. It increases the parasympathetic modulation and/or reduces the sympathetic component. Thus, slow breathing is proposed as an alternative therapy to reduce blood pressure [22].

Based on our results, the SDNN index was not altered in the control protocol, while it significantly distorted in the initial $5 \mathrm{~min}$ after the PCM in the slow breathing protocol, indicating that slow breathing increased its responses. The SDNN index corresponds to global heart rate modulation [7]. However, it does not provide information regarding whether the sympathetic or parasympathetic component of the ANS is modified.

The parasympathetic cardiac regulation is represented by RMSSD and pNN50 indices in the time domain and, by the HF index in the frequency domain [23]. It is well documented in the literature that those indices are reduced at standing compared to seated $[24,25]$. We reported reduced values of all aforesaid indices at orthostatic position compared to seated in the control and slow breathing protocols. More intense responses were observed in the slow breathing protocol, supporting that slow breathing at 6 cycles per minutes intensifies HRV responses to PCM.

Slow breathing has received consideration since it has been used as an alternative or complementary therapy for cardiovascular disorders. A previous study reported that slow breathing reduced arterial blood pressure and improved baroreflex sensitivity in essential hypertensive patients [26]. Another study found that slow breathing at the same rate used in our analysis decreased chemoreflex responses induced by hypercapnia and hypoxia [27].

With regards to the sympathetic cardiac responses to PCM, the spectral analysis indicated that the LF/HF ratio presented significant responses $0-5 \mathrm{~min}$ after the volunteers stood up in the slow breathing protocol. Alternatively, the same index did not change in the control protocol, reinforcing the acute effects of slow breathing on cardiac autonomic responses.

Previous studies illustrated the involvement of slow breathing in sympathetic responses. Fonoberova et al. [28] performed a specific computational physiological technique to model cardiac autonomic regulation during slow breathing in humans. The authors reported beneficial effects of slow breathing on sympathetic component of the baroreflex. As follows, our study provides additional effects of slow breathing, since it improved cardiac sympathetic and parasympathetic reactivation induced by a specific autonomic test. 
The higher cardiac autonomic responses to PCM caused by slow breathing observed in our study may be explained by its effects on controlling physiological arousal. Wells et al. [4] investigated the influence of acute slow breathing on cardiac autonomic modulation and anxiety. The authors reported that positive efficacy of slow breathing on HRV values in response to stress followed by reduction in anxiety levels in anxious subjects. Overall, we may hypothesize that slow breathing influenced cardiac autonomic responses to PCM due to its interaction with physiological stress reactivity.

Alterations in HRV patterns represents an early and sensitive indicator of cardiovascular events. Increased HRV corresponds to good adaptation of the ANS and, it is related to a healthy individual with well-organized autonomic activity [29]. Moreover, attenuated HRV responses to postural changes maneuver were found in patients with cardiovascular disorders [30,31].

Subjects with autonomic failure presented impaired cardiac autonomic responses to orthostatism, inducing postural hypotension caused by injury in parasympathetic activity [32]. Orthostatic hypotension is considered the hallmark of autonomic dysfunction, which was related to hypertension and pathologic ventricular hypertrophy [33]. Moreover, orthostatic hypotension was also designated as a risk factor for developing atrial fibrilation, coronary disorders, stroke, heart failure and chronic kidney disease [34-36]. Similarly, the slow breathing protocol applied in our study acutely improved autonomic responses, supporting its beneficial effects on ANS.

We may propose a hypothesis to explain the increase in HRV reactivity to postural change. It is feasible that previous parasympathetic activation induced by slow breathing intensified cardiac autonomic responses to PCM. The brainstem plays an important role in autonomic regulation. Areas such as surrounding the fourth cerebral ventricle - related to the integration between cardiopulmonary and baroreflex mechanisms. [37] In this circumstance we suggest that slow breathing changes baroreflex responses to PCM, leading to amplification of HRV responses to this maneuver.

Our results highlight the technique of slow breathing as an alternative or complementary therapy. It acutely increased parasympathetic cardiac regulation and improved the autonomic adaptation to respond to internal stimuli.

\section{Conclusion}

Slow breathing intensified cardiac autonomic responses induced by PCM from seated to orthostatic position. Our data supports the beneficial acute effects of slow breathing on HRV responses to a specific autonomic test.

\section{Acknowledgments}

The authors declare that there is no conflict of interests regarding the publication of this article. The study received financial support from CNPq (Grant no. 29360).

\section{References}

[1] M.R. Altena, N. Kleefstra, S.J. Logtenberg, K.H. Groenier, S.T. Houweling, H.J. Bilo, Effect of device-guided breathing exercises on blood pressure in patients with hypertension: a randomized controlled trial, Blood Press. 18 (5) (2009) 273-279.

[2] T. Drozdz, G. Bilo, D. Debicka-Dabrowska, et al., Blood pressure changes in patients with chronic heart failure undergoing slow breathing training, Blood Press. (2015 Oct 29) 1-8 [Epub ahead of print].

[3] A. Chaddha, Slow breathing and cardiovascular disease, Int. J. Yoga 8 (2) (2015) 142-143.

[4] R. Wells, T. Outhred, J.A. Heathers, D.S. Quintana, A.H. Kemp, Matter over mind: a randomised-controlled trial of single-session biofeedback training on performance anxiety and heart rate variability in musicians, PLoS One 7 (10) (2012) e46597.

[5] D. Paprika, Z. Gingl, L. Rudas, E. Zöllei, Hemodynamic effects of slow breathing: does the pattern matter beyond the rate? Acta Physiol. Hung. 101 (3) (2014) 273-281.

[6] Q. Chang, R. Liu, Z. Shen, Effects of slow breathing rate on blood pressure and heart rate variabilities, 25, Int. J. Cardiol. 169 (1) (2013) e6-8.

[7] Task Force of the European Society of Cardiology and the North American Society of Pacing and Electrophysiology, Heart rate variability: standards of measurement, physiological interpretation and clinical use, Circulation 93 (1993) 1043-1065.

[8] V.E. Valenti, The use of heart rate variability for research, J. Hum. Growth Dev. 25 (2015) 137-140.

[9] L.C. Vanderlei, C.M. Pastre, R.A. Hoshi, T.D. Carvalho, M.F. Godoy, Basic notions of heart rate variability and its clinical applicability, Rev. Bras. Cir. Cardiovasc. 24 (2) (2009) 205-217.

[10] C.N. Joseph, C. Porta, G. Casucci, et al., Slow breathing improves arterial baroreflex sensitivity and decreases blood pressure in essential hypertension, Hypertension 46 (4) (2005) 714-718.

[11] C.H. Pinheiro, R.A. Medeiros, D.G. Pinheiro, Mde J. Marinho, Spontaneous respiratory modulation improves cardiovascular control in essential hypertension Arq, Bras. Cardiol. 88 (6) (2007) 651-659.

[12] D. Ziegler, Diabetic cardiovascular autonomic neuropathy: prognosis, diagnosis and treatment, Diabetes Metab. Rev. 10 (1994) 339-383.

[13] C.U. Jones, B. Sangthong, O. Pachirat, D.A. Jones, Slow breathing training reduces resting blood pressure and the pressure responses to exercise, Physiol. Res. 24 (2015) [Epub ahead of print].

[14] R. Barbosa, NTd Silva, FMd Azevedo, C.M. Pastre, L.C.M. Vanderlei, Comparison of Polar ${ }^{\mathbb{R}}$ RS800G3 ${ }^{\mathrm{TM}}$ heart rate monitor with Polar ${ }^{\mathbb{B}}$ S810i ${ }^{\mathrm{TM}}$ and electrocardiogram to obtain the series of RR intervals and analysis of heart rate variability at rest, Clin. Physiol. Funct. Imaging (2014). http://onlinelibrary.wiley. com/doi/10.1111/cpf.12203/epdf.

[15] R.B. Blackman, J.W. Tukey, The Measurement of Power Spectra from the Point of View of Communication Engineering, 1958. Dover, New York1958.

[16] F.M. Vanderlei, L.C. Vanderlei, V.E. Valenti, L.C. Abreu, Heart rate variability in healthy adolescents at rest. In: V Congresso Internacional de Saúde da Criança e do Adolescente, 2013, São Caetano, J. Hum. Growth Dev. 23 (1) (2013), $23-23$.

[17] M.P. Tarvainen, J.-P. Niskanen, J.A. Lipponen, P.O. Ranta-Aho, P.A. Karjalainen, Kubios HRV-heart rate variability analysis software, Comput. Methods Programs Biomed. 113 (1) (2014) 210-220.

[18] J. Hayano, S. Mukai, M. Sakakibara, A. Okada, K. Takata, T. Fujinami, Effects of respiratory interval on vagal modulation of heart rate, Am. J. Physiol. Heart Circ. Physiol. 267 (1994) 33-40.

[19] G.G. Berntson, J.T. Cacioppo, K. Quigley, Respiratory sinus arrhythmia: autonomic origins, physiological mechanisms, and psychophysiological implications, Psychophysiology 30 (2) (1993) 183-196.

[20] G.E. Billman, Heart rate variability-a historical perspective, Front. Physiol. 2 (2011 Nov 29) 86.

[21] M.S. Reis, R. Arena, B. Archiza, C.F. de Toledo, A.M. Catai, A. Borghi-Silva, Deep breathing heart rate variability is associated with inspiratory muscle weakness in chronic heart failure, Physiother. Res. Int. 19 (1) (2014) 16-24.

[22] K.R. Mahtani, D. Nunan, C.J. Heneghan, Device-guided breathing exercises in the control of human blood pressure: systematic review and meta-analysis, J. Hypertens. 30 (5) (2012) 852-860.

[23] L.C. Vanderlei, C.M. Pastre, R.A. Hoshi, T.D. Carvalho, M.F. Godoy, Basic notions of heart rate variability and its clinical applicability, Rev. Bras. Cir. Cardiovasc. 24 (2) (2009) 205-217.

[24] A.C. de Souza, J.R. Cisternas, L.C. de Abreu, et al., Fractal correlation property of heart rate variability in response to the PCM in healthy women, Int. Arch. Med. 15 (7) (2014) 25.

[25] B.C. de Castro, H.L. Guida, A.L. Roque, et al., Previous exposure to musical auditory stimulation immediately influences the cardiac autonomic responses to the PCM in women, 14, Int. Arch. Med. 6 (1) (2013) 32.

[26] C.N. Joseph, C. Porta, G. Casucci, et al., Slow breathing improves arterial baroreflex sensitivity and decreases blood pressure in essential hypertension, Hypertension 46 (4) (2005) 714-718.

[27] L. Bernardi, A. Gabutti, C. Porta, L. Spicuzza, Slow breathing reduces chemoreflex response to hypoxia and hypercapnia, and increases baroreflex sensitivity, J. Hypertens. 19 (12) (2001) 2221-2229.

[28] M. Fonoberova, I. Mezić, J.F. Buckman, et al., A computational physiology approach to personalized treatment models: the beneficial effects of slow breathing on the human cardiovascular system, 1, Am. J. Physiol. Heart Circ. Physiol. 307 (7) (2014) H1073-H1091.

[29] A. Cherkas, R. Zhuraev, A marked decrease in heart rate variability in Marfan syndrome patients with confirmed FBN1 mutations, Cardiol. J. (2015 Oct 27), http://dx.doi.org/10.5603/CJ.a2015.0076.

[30] G. Sannino, P. Melillo, S. Stranges, G.D. Pietro, L. Pecchia, Blood pressure drop prediction by using HRV measurements in orthostatic hypotension, J. Med. Syst. 39 (11) (2015) 292.

[31] V. Milazzo, C. Di Stefano, A. Milan, et al., Cardiovascular complications in patients with autonomic failure, Clin. Auton. Res. 25 (3) (2015) 133-140.

[32] X.H. Fan, Y. Wang, K. Sun, et al., Disorders of orthostatic blood pressure response are associated with cardiovascular disease and target organ damage 
in hypertensive patients, Am. J. Hypertens. 23 (8) (2010) 829-837.

[33] N. Franceschini, K.M. Rose, B.C. Astor, D. Couper, S. Vupputuri, Orthostatic hypotension and incident chronic kidney disease: the atherosclerosis risk in communities study, Hypertension 56 (6) (2010) 1054-1059.

[34] M.L. Eigenbrodt, K.M. Rose, D.J. Couper, D.K. Arnett, R. Smith, D. Jones, Orthostatic hypotension as a risk factor for stroke: the atherosclerosis risk in communities (ARIC) study, 1987-1996, Stroke 31 (10) (2000) 2307-2313.

[35] A. Fedorowski, G. Engstrom, B. Hedblad, O. Melander, Orthostatic hypotension predicts incidence of heart failure: the Malmo preventive project, Am. J Hypertens. 23 (11) (2010) 1209-1215.

[36] J.R. Cisternas, V.E. Valenti, M.A. Sato, et al., The effects of catalase inhibition into the fourth cerebral ventricle on the Bezold-Jarisch reflex in spontaneously hypertensive rats, J. Integr. Neurosci. 10 (4) (2011) 475-487.

[37] V.E. Valenti, L.C. Abreu, M.A. Sato, C.A.T.Z. Ferreira, (3-amino-1,2,4-triazole) injected into the fourth cerebral ventricle influences the Bezold-Jarisch reflex in conscious rats, Clinics 65 (12) (2010) 1339-1343. 